\title{
The heritability of carrot resistance to fungal diseases of Alternaria and Fusarium genus
}

\author{
Balashova I.T. ${ }^{1 *}$, Sokolova L.M. ${ }^{2}$, Sirota S.M. ${ }^{1}$ \\ ${ }^{1}$ Federal Scientific Vegetable Center, Moscow region, Russia \\ ${ }^{2}$ Branch of the Federal Scientific Vegetable Center, Moscow region, Russia \\ *email: balashova56@mail.ru
}

Alternaria dauci, Alternaria radicina and pathogenic species from Fusarium genus are the most damaging diseases affecting carrots (Daucus carota L.). A. dauci causes leaf blight and premature death of leaves. A. radicina causes rots 50-70\% of carrot roots during storage. Pathogenic species of Fusarium genus cause the tracheomycosis wilt, retardation growth of the plant, root and stem rots, "black leg" of seedlings. The goal of study is to obtain $\mathrm{F}_{1}$-hybrids of carrots with field resistance to the group pathogens of Alternaria and Fusarium genus. Obtaining carrot varieties and hybrids with group resistance to main pathogens is very difficult task, which is complicated by the fact that carrot resistance to fungal diseases has the polygenic control. The heritability of resistance-trait to a group of pathogens by $\mathrm{F}_{1}$-hybrids is conveniently determined with the heritability coefficient $h^{2}$, which is the only indicator determining the inclusion of genetic component to the overall variability of the trait, even at the genomic era. Resistance the basic lines of carrot to fungi of Alternaria and Fusarium genus has been studied with step by step evaluation, using several types of hard artificial and natural infection backgrounds. Tolerant samples were selected and they were used in crossings. $\mathrm{F}_{1}$-hybrids have been obtained and they have been evaluated at artificial and natural backgrounds.

Results:

1. One tolerant male form \# 1268 and 6 female forms with weak sensitivity to fungi of Alternaria and Fusarium genus were selected.

2. These forms were used in crossings and $F_{1}$-hybrids have been obtained.

3. $F_{1}$-hybrids were evaluated at artificial and natural infection backgrounds.

4. Analysis the heritability of carrot resistance to fungi of Alternaria and Fusarium genus identified, that carrot resistance to group pathogens didn't inherited by mother's type.

5. Using tolerant carrot line \# 1268 in crossings as the male form and 6 female forms with weak sensitivity to fungi of Alternaria and Fusarium genus increased significantly the percent of tolerant plant in $\mathrm{F}_{1}$-hybrid population evaluated under artificial infection. 